\title{
ATUAÇÃO FISIOTERAPÊUTICA PARA REDUÇÃO DO QUADRO ÁLGICO NO TRABALHO DE PARTO ATIVO
}

Physiotherapeutic action for reduction of table painful assets in labor

Joice de Abreu Brandolfi ${ }^{1}$, Kelen Gava Duminelli ${ }^{2}$, Elisa da Silva Bobsin ${ }^{2}$, Kristian Madeira $^{3}$, Robson Pacheco ${ }^{3}$, Ariete Inês Minetto ${ }^{4}$

${ }^{1}$ Curso de Fisioterapia da Universidade do Extremo Sul Catarinense (UNESC) Criciúma (SC), Brasil.

${ }^{2}$ Fisioterapeuta graduada pelo Curso de Fisioterapia da Universidade do Extremo Sul Catarinense (UNESC) - Criciúma (SC), Brasil.

${ }^{3}$ Docente do Curso de Fisioterapia da Universidade do Extremo Sul Catarinense (UNESC) - Criciúma (SC), Brasil.

${ }^{4}$ Coordenadora Adjunta e Docente do Curso de Fisioterapia da Universidade do Extremo Sul Catarinense (UNESC) - Criciúma (SC), Brasil.

\section{Endereço para correspondência:}

Ariete Inês Minetto

Departamento de Fisioterapia - UNESC

Av. Universitária, 1105, Bairro Universitário, Bloco S, $1^{\circ}$ andar, sala 8

CEP - 88806-000 - Criciúma - SC

Email: ariete@unesc.net 


\section{Resumo}

A Fisioterapia no trabalho de parto, como forma de assistência à saúde da mulher, apresenta-se em ascensão. Trata-se de uma intervenção no ambiente onde se encontra a gestante, que necessita de cuidados e orientações sobre posicionamento, educação para o parto e medidas de alívio em caso de dor. 0 estudo teve como objetivo a promoção da redução do quadro álgico através da intervenção fisioterapêutica em gestantes em trabalho de parto ativo do Centro Obstétrico de um Hospital do Sul de Santa Catarina (SC). A amostra foi dividida em dois grupos sendo o Grupo 1 (G1), que recebeu a aplicação de um protocolo fisioterapêutico, baseado nas condutas de terapia manual e adoção de posturas verticais e o Grupo 2 (G2), que compôs o grupo placebo, o qual recebeu apenas afago, ou seja, simulação de tratamento. Participaram da pesquisa 16 gestantes, com idade média de 24,69 anos ( $\pm 5,49$ ). Para a graduação da dor foi utilizada a Escala Visual Analógica (EVA). Nas aplicações da escala EVA durante o tempo de trabalho de parto ativo, percebeu-se que em média, a dor das gestantes do G1 foi menor que a das gestantes do G2 a partir de 60 minutos. Possibilitando a conclusão de que, a abordagem fisioterapêutica junto à equipe de centros obstétricos é benéfica, atuando em orientações quanto a mobilidade adequada à gestante, aderindo o uso de posturas verticais bem como o uso de técnicas de terapia manual, influenciando de maneira positiva na fase ativa do trabalho de parto, diminuindo a dor e o tempo do trabalho de parto, demonstrando a relevância desse estudo.

Palavras-chave: Fisioterapia. Parto Normal. Dor.

\section{Abstract}

Physiotherapy in labor, as a form of health care of women, presents on the rise. This is an intervention in the environment where the pregnant woman who needs care and guidance about placement, education for childbirth and pain relief measures. To promote the reduction of pain through physiotherapy intervention in pregnant women in active labor the obstetric center of a Hospital of Southern Santa Catarina (SC). The sample was divided into two groups with the G1, which received application of a physical therapy protocol, based on Manual Therapy behaviors and adopting postures Vertical and G2, which composed the placebo group, which received only cuddle, that is, treatment simulation. 16 pregnant women participated in the survey, with a mean age of 24.69 years $( \pm 5.49)$. For pain graduation was used Visual Analog Scale (VAS). In applications VAS scale during the time of active labor, it was noticed that on average, pain in pregnant women G1 was lower than that of women in the G2 from 60 minutes. We conclude that the physiotherapist is critical piece together the team of obstetric centres, guiding the proper mobility to pregnant 
women, adhering the use of Vertical postures and use of manual therapy techniques, influencing positively the active phase of labor, reducing pain and the time of labor, demonstrating the relevance of this study.

Keywords: Physiotherapy. Normal childbirth. Pain.

\section{INTRODUÇÃO}

O trabalho do fisioterapeuta na assistência à saúde da mulher encontrase em escala crescente. Medidas de alívio da dor e o acompanhamento da gestante em trabalho de parto ativo têm sido temáticas presentes em pesquisas e abordagens e podem ser aplicadas pelo terapeuta sob forma de instruções de posicionamento, educação para o parto e o uso de terapia manual e adoção de posturas verticais ${ }^{1,2}$.

Mesmo nos tempos atuais o momento do parto é caracterizado por sentimentos de angústia, medo e de desorientação pelas parturientes, já que as mulheres, de modo geral, não estão preparadas para o enfrentamento dessa etapa. A Fisioterapia propõe que a mulher se prepare e conscientize-se sobre a necessidade de se manter calma e relaxada durante todo o trabalho de parto. A fim de viabilizar essa possibilidade, o fisioterapeuta poderá lançar mão de métodos e técnicas que permitam vencer de maneira natural a dor do parto e ao mesmo tempo potencialize a musculatura, a consciência para o controle e a coordenação motora, com intuito de proporcionar a diminuição da necessidade do uso de medicamentos analgésicos e anestésicos que podem causar efeitos deletérios à mãe e ao feto durante o processo de parturição ${ }^{3,4}$.

Ao início do trabalho de parto, é comum que as gestantes sintam dores advindas das contrações, presentes no primeiro e no segundo estágio. A evolução do trabalho de parto é mensurada pela cervicodilatação uterina, por meio do toque vaginal, sendo que a progressão da dilatação cervical acelera-se a partir dos $4 \mathrm{~cm}$, caracterizando a fase ativa do trabalho de parto, quando a velocidade da dilatação cervical se dá em torno de $1 \mathrm{~cm}$ por hora ${ }^{1,4,5}$.

Para Castro et al. ${ }^{6}$ no estágio I, que compreende o período de dilatação e afinamento do colo uterino conforme o feto desce no canal de parto, há a presença do estresse emocional, bem como da dor, que se intensifica conforme o término do primeiro estágio. No estágio I a dor é visceral, não localizada, difusa e intermitente, o 
que difere do estágio seguinte, o estágio II, que é o de expulsão do feto, onde a dor tem um componente somático, se manifesta de forma intensa, localizada e contínua. A dor sentida pela mulher nesse período é uma sensação individual e multifatorial, podendo estar associada à lesão tecidual e sofrer influências socioculturais, psicológicas, econômicas e do ambiente onde são manifestadas, devendo ser respeitada e não subestimada ${ }^{6,7}$.

A presença do fisioterapeuta no acompanhamento do trabalho de parto ainda não se constitui como uma prática estabelecida na sociedade contemporânea, e não está inclusa no sistema de saúde pública, porém, esse profissional dispõe de recursos não farmacológicos para o alívio da dor da gestante no estágio I do trabalho de parto. Dentre esses recursos pode-se destacar a terapia manual e a adoção de posturas verticais ${ }^{1,8}$.

Durante o trabalho de parto, a parturiente requer mobilidade pélvica e uso intensivo da musculatura do abdômen, do períneo e do diafragma respiratório. Portanto, o fisioterapeuta está apto para lidar com as múltiplas alterações musculoesqueléticas que ocorrem em resposta à gravidez, por estudar os movimentos das articulações do corpo humano e as implicações de sua biomecânica muscular quando aplicada à prática clínica. Além de atuar na otimização da fisiologia e da anatomia humana, auxiliando as gestantes na adoção de posturas no trabalho de parto, na contração e principalmente no relaxamento da musculatura dorsal, do assoalho pélvico e do canal vaginal, podendo contribuir ativamente no atendimento à parturiente $\mathrm{e}^{9,10,11,12}$.

Sendo assim, a intervenção fisioterapêutica na assistência obstétrica de baixo risco, como parte da rotina da equipe interdisciplinar, valoriza a responsabilidade da gestante no processo, por meio do uso ativo do próprio corpo, através de mobilidade corporal, bem como preconiza a Organização Mundial da Saúde desde $1996^{13}$.

O estudo teve como objetivo, observar através da Escala Visual Analógica (EVA) a possível redução da dor durante o trabalho de parto ativo, através da aplicação da intervenção fisioterapêutica, bem como analisar se a fisioterapia interfere no atendimento das parturientes junto às equipes de centros obstétricos. 
O estudo classifica-se como intervencional, sendo um ensaio de campo randomizado (14). Foi aprovado pelo Comitê de Ética (CEP) em humanos da UNESC (SC) sob o parecer № 343.980/2013 e posteriormente pelo Comitê de Ética do hospital onde o estudo foi realizado, sob o parecer № 371.843/2013. No período, foram abordadas 17 gestantes em trabalho de parto ativo, sendo que todas foram informadas sobre o procedimento do estudo e assinaram um Termo de Consentimento Livre e Esclarecido (TCLE). Uma das gestantes se negou a participar da pesquisa, sendo então excluída da amostra, bem como as gestantes de alto risco, multifetais, em trabalho de parto latente ou com indicação de parto cesárea. A participação se deu por conveniência e as parturientes foram submetidas à pesquisa no Centro Obstétrico de um Hospital do Sul de Santa Catarina.

Para a coleta de dados foi utilizado o instrumento de avaliação, contendo questões sobre dados pessoais e do histórico gestacional; Prontuário eletrônico do Centro Obstétrico, para verificação da evolução do trabalho de parto; Escala Visual Analógica (EVA), para graduação da dor das gestantes durante o procedimento, sendo que de zero a dois considera-se dor leve, de três a sete dor moderada e de oito a dez dor intensa ${ }^{15}$.

Nesse estudo trabalhou-se com duas amostras de gestantes em trabalho de parto ativo, que foram submetidas à aplicação de um Protocolo de Intervenção Fisioterapêutica, validado por três profissionais fisioterapeutas, e um Placebo. $O$ grupo 1 (G1) recebeu aplicação de Protocolo Fisioterapêutico baseado nas condutas de terapia manual, que incluíram deslizamento superficial e profundo associados à massoterapia, e adoção de posturas verticais, sendo que estas posturas apresentam vantagens quando se leva em consideração o ponto de vista gravitacional. Ao executadas promovem uma retificação do canal de parto e alinhamento do feto, o que facilita o desprendimento fetal, e que pode contribuir na assistência ao parto ${ }^{1,9}$. Já o grupo 2 (G2) que compôs o grupo Placebo, recebeu apenas afago, ou seja, simulação de tratamento sem propriedades terapêuticas.

Os procedimentos terapêuticos foram realizados a partir da aceitação da gestante e preenchimento do instrumento de avaliação, logo depois que as pesquisadoras verificavam a ordem de internação da paciente para fazer então a 
escolha do protocolo a ser aplicado. A primeira gestante recebeu a Intervenção Fisioterapêutica, a segunda o Placebo e assim sucessivamente, fazendo com que houvesse uma intercalação entre os protocolos. Para a aplicação do protocolo de Intervenção Fisioterapêutica foram utilizados alguns materiais como: óleo hipoalergênico da marca Extratos da Terra (terapia manual) e de uma bola suíça da marca Mercur, almofadas ou travesseiros e uma cadeira (posturas verticais). No protocolo Placebo a gestante recebeu apenas afagos no dorso, com a paciente em decúbito lateral esquerdo, para simulação de tratamento.

Ao iniciar a aplicação do protocolo com a gestante em atendimento, foi graduada a dor pela EVA de $30 \mathrm{em} 30$ minutos, até que o trabalho de parto chegasse ao final, para verificar se com a aplicação do protocolo proposto, haveria redução do quadro álgico. Vale salientar que as gestantes foram atendidas conforme o tempo de trabalho de parto individual, considerando que estas apresentam tempo de evolução diferenciado, bem como sintomatologias distintas, sendo que o protocolo foi ajustado de acordo com o tempo das contrações e das necessidades de cada uma das gestantes. No momento em que se encerrou o trabalho de parto, as pesquisadoras acompanharam as gestantes que evoluíram para o parto normal, para participação no procedimento na sala de parto, a fim de que não houvesse o corte do vínculo entre gestante e fisioterapeuta, adquirido durante o atendimento. Nos partos que evoluíram para cesárea, as pesquisadoras não fizeram o acompanhamento do procedimento, em função de normatização do Centro Obstétrico. Após o nascimento do bebê, as pesquisadoras finalizaram o atendimento fazendo à gestante a seguinte pergunta aberta: "No seu ponto de vista, a intervenção fisioterapêutica contribuiu com a evolução do parto? De que forma?". Após, foi entregue a gestante um material contendo orientações para o puerpério, contendo informações sobre posturas a serem adotadas, amamentação e cuidados com o bebê.

Para análise dos dados e avaliação foi utilizada a estatística descritiva (média e desvio padrão, mediana e amplitude interquartil e frequências $e$ porcentagens). Para a comparação do escore médio obtido pela aplicação da EVA entre os grupos de Intervenção Fisioterapêutica (G1) e Placebo (G2), utilizou-se o teste $t$ de Student, e dentro dos grupos nos diferentes tempos de observação o teste de Friedman. O software utilizado para análise foi o IBM Statistical Package for the 
Artigo Original

Atenção à Saúde

Social Sciencies (SPSS) versão 20.0. Os testes estatísticos foram realizados com um nível de significância $\alpha=0,05$ e um intervalo de confiança de $95 \%$.

A comparação da média das variáveis quantitativas entre os grupos protocolo e placebo foi realizada por meio da aplicação do teste t de Student quando apresentavam distribuição normal e pelo teste $U$ de Mann-Whitney quando não apresentavam esse tipo de distribuição. A homocedasticidade das variâncias foi averiguada por meio da aplicação do teste de Levene e a normalidade foi averiguada por meio do teste de Shapiro-Wilk. A verificação da existência de associação entre as variáveis qualitativas entre $\mathrm{G} 1$ e G2, foi realizada por meio da aplicação dos testes qui-quadrado de Pearson e exato de Fisher.

\section{RESULTADOS}

$\mathrm{Na}$ tabela 1 descrevem-se os dados quantitativos e qualitativos referentes à amostra da pesquisa, onde as gestantes que fizeram parte desse estudo compõem uma média de idade de 24,69 anos $( \pm 5,49)$, sendo que o $\mathrm{G} 1$ apresentou uma média de idade de 23,70 anos $( \pm 5,81)$ e no $G 2$ a média obtida foi de 26,33 anos $( \pm 4,97)$. Do total da amostra, $62,5 \%$ eram primigestas, totalizando 6 gestantes.

Quando relacionamos o ganho de peso durante a gestação, as mulheres apresentaram em média um aumento de $16,75 \mathrm{~kg}( \pm 4,90)$ em $100 \%$ da amostra, sendo que o G1 apresentou $17,20 \mathrm{~kg}( \pm 5,59)$ e o G2 $16,00 \mathrm{~kg}( \pm 3,85)$.

Sobre o tempo total de internação no Centro Obstétrico, na comparação entre os grupos, o G1 apresentou tempo total de 377,50min $(193,25$ - 506,25) e o G2 de 544,00min (409,50 - 772,50). Durante a atuação fisioterapêutica o G1 obteve um tempo total de $165,00 \mathrm{~min}(115,00-346,25)$ e o G2 de $155,00 \mathrm{~min}(107,50$ $277,75)$.

Descreveram-se ainda os dados referentes às características gestacionais como: gestação planejada; gestações anteriores; qual o tipo de parto anterior e a evolução do parto atual; realizou alguma intervenção ou manobra, bem como o tipo de manobra; fez uso ou não de medicamento durante o trabalho de parto. 
Artigo Original

Atenção à Saúde

Para a Tabela 1, a qual aborda os dados quantitativos e qualitativos, os resultados encontrados não apresentaram significância estatística, considerando $p \geq$ 0,05 .

Tabela 1. Características da Amostra

\begin{tabular}{|c|c|c|c|}
\hline & \multicolumn{2}{|c|}{ Média \pm DP ou n (\%) } & \multirow[b]{2}{*}{ valor-p } \\
\hline & Protocolo & Placebo & \\
\hline Idade (anos) & $23,70 \pm 5,81$ & $26,33 \pm 4,97$ & 0,372 \\
\hline Idade gestacional & $39,54 \pm 0,98$ & $39,83 \pm 0,75$ & 0,542 \\
\hline Aumento de Peso & $17,20 \pm 5,59$ & $16,00 \pm 3,85$ & 0,652 \\
\hline Gestações anteriores* & $0,00(0,00-1,00)$ & $0,00(0,00-1,00)$ & 0,703 \\
\hline Internação (min) * & $377,50(193,25-506,25)$ & $544,00(409,50-772,50)$ & 0,083 \\
\hline $\begin{array}{l}\text { Atuação Fisioterapêutica } \\
(\min )^{*}\end{array}$ & $165,00(115,00-346,25)$ & $155,00(107,50-277,75)$ & 0,625 \\
\hline \multicolumn{4}{|l|}{ Gestação Planejada } \\
\hline Sim & $4(40,0)$ & $4(66,7)$ & 0,608 \\
\hline Não & $6(60,0)$ & $2(33,3)$ & \\
\hline \multicolumn{4}{|l|}{$\begin{array}{l}\text { Teve gestações } \\
\text { anteriores }\end{array}$} \\
\hline Sim & $4(40,0)$ & $2(33,3)$ & 1,000 \\
\hline Não & $6(60,0)$ & $4(66,7)$ & \\
\hline \multicolumn{4}{|l|}{ Partos Anteriores } \\
\hline Primeiro Parto & $6(60,0)$ & $4(66,7)$ & 0,465 \\
\hline Normal & $3(30,0)$ & $1(16,7)$ & \\
\hline Cesárea & $0(0,0)$ & $1(16,7)$ & \\
\hline Aborto & $1(10,0)$ & $0(0,0)$ & \\
\hline \multicolumn{4}{|l|}{ Evolução do Parto } \\
\hline Normal & $6(60,0)$ & $4(66,7)$ & 1,000 \\
\hline Cesárea & $4(40,0)$ & $2(33,3)$ & \\
\hline \multicolumn{4}{|l|}{ Realizou Intervenção } \\
\hline Sem Intervenção & $3(30,0)$ & $3(50,0)$ & 0,319 \\
\hline Episiotomia & $4(40,0)$ & $3(50,0)$ & \\
\hline Laceração & $3(30,0)$ & $0(0,0)$ & \\
\hline \multicolumn{4}{|l|}{ Manobra de Kristeller } \\
\hline Sim & $1(10,0)$ & $1(16,7)$ & 0,319 \\
\hline Não & $9(90,0)$ & $5(83,3)$ & \\
\hline \multicolumn{4}{|l|}{ Fez uso de Ocitocina } \\
\hline Sim & $10(100,0)$ & $4(66,7)$ & 0,125 \\
\hline Não & $0(0,0)$ & $2(33,3)$ & \\
\hline \multicolumn{4}{|l|}{ Fez uso de Misoprostol } \\
\hline Sim & $1(10,0)$ & $3(50,0)$ & 0,118 \\
\hline Não & $9(90,0)$ & $3(50,0)$ & \\
\hline
\end{tabular}


Fez uso de Dipirona

Sim

Não

$10(100,0)$

Fonte: Dados da Pesquisa

Nota: ${ }^{*} \mathrm{AIQ}=$ Amplitude Interquartil

Legenda: DP = Desvio Padrão

Conforme a Figura 1, que comparou a EVA entre o G1 e o G2, a amostra sugere a redução considerável da dor, mesmo que não observável a sua significância estatística em relação aos grupos quando $p \geq 0,05$. Notou-se nos iniciais $30 \mathrm{~min}$ de trabalho de parto ativo, um alto escore na intensidade da dor.

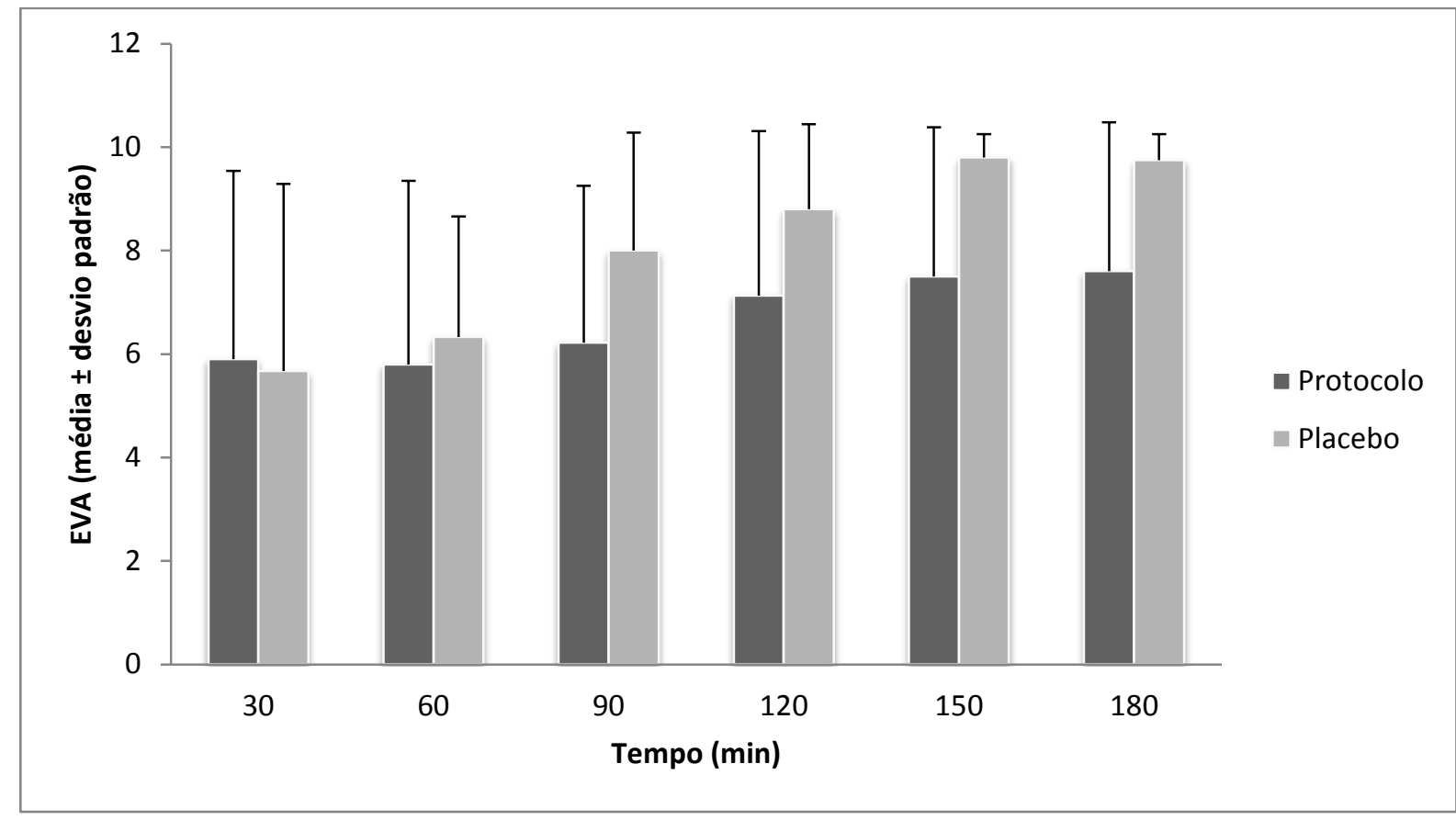

Figura 1. Escala Visual Analógica (EVA)

Nota: Dados da pesquisa.

\section{DISCUSSÃO}

Inicialmente o presente estudo objetivou a observação da possibilidade de redução da dor das gestantes em trabalho de parto ativo, por meio da Escala EVA, através da aplicação dos Protocolos de Intervenção Fisioterapêutica e Placebo, sendo observados fatores alheios aos pesquisados que podem influenciar no quadro álgico durante o processo do trabalho de parto. 
Tendo em vista tratar-se de uma amostra pequena, composta por 16 gestantes, na análise da dor durante a aplicação dos Protocolos, ao utilizarmos a EVA, verificamos que não houve diferença estatística significativa entre os grupos G1 e G2, mesmo percebendo a diminuição da dor do G1 à partir dos 60min de intervenção fisioterapêutica. Observou-se que a dor permaneceu num limiar fisiológico onde a intensidade da percepção dolorosa é individual e multidimensional, ou seja, amplamente variável de acordo com as expectativas de cada gestante. Assim como a escala utilizada pelas pesquisadoras, existem ainda vários métodos e instrumentos disponíveis para mensurar a dor, os quais são da mesma forma, considerados subjetivos e de difícil quantificação ${ }^{7,16,17}$.

Estudos comprovam que a dor pode variar conforme o suporte e as orientações recebidas pelas gestantes no momento do trabalho de parto ativo, isto se dá pelo uso de recursos não farmacológicos de alívio desta dor, tais como a presença de um acompanhante, ambiente calmo, banhos de chuveiro e técnicas de Terapia Manual, reforçando a presença do Fisioterapeuta durante o trabalho de parto, com o intuito de diminuir a tríade medo, tensão e dor. Isso se mostrou evidente durante o relato (Entrevistada № 08 ) às pesquisadoras que expõe que "Foi ótimo, realmente muito bom. Como eu fiquei tensa, a presença de vocês me acalmou. Ajudou muito no equilíbrio da respiração. $O$ acompanhamento direto de vocês ajudou na evolução do meu parto" 7,18.

O número de partos anteriores também constituiu um importante fator para as autoras, salvo que $62,5 \%$ das gestantes da amostra eram primigestas, o que interferiu na expectativa do que estava por acontecer, visto que essas gestantes não tinham ciência da dor do trabalho de parto, referindo ser esta maior que a esperada. Em estudo realizado em 2010, constatou-se que gestantes primigestas que passaram por curso de preparação para o parto apresentaram menores escores de dor comparadas àquelas que não se submeteram à tal preparação ${ }^{19}$.

Durante a pesquisa verificou-se que as parturientes com idade próxima aos 18 anos com gestação não planejada e sem preparação para o parto, produziram uma gravidez permeada por transtornos físicos e emocionais, contudo a outra metade, que planejou sua gravidez, são mulheres com preparação adequada para exercer uma das mais importantes funções femininas, a de gerar vidas. $O$ planejamento familiar apresenta-se importante no enfrentamento deste momento 
para as gestantes, tornando-se imprescindível o suporte familiar, social e da equipe de saúde, pois essas mulheres quando orientadas e preparadas sentem-se gratificadas por cooperarem durante o processo de parto, mudando completamente sua visão à respeito do parto, antes muito temido $4,7,20,21$.

As gestantes avaliadas em relação ao aumento de peso durante a gestação estão acima da média proposta, onde segundo a Organização Mundial da Saúde (OMS), o aumento ideal de peso durante a gestação depende da condição da mulher antes da gravidez. Se ela estiver abaixo dos índices normais, é aceitável que engorde entre 12 e $18 \mathrm{~kg}$. Para as mães com sobrepeso, são tolerados entre $7 \mathrm{e}$ $11,5 \mathrm{~kg}$ a mais durante os 9 meses. E para aquelas que se encontram com peso adequado, recomenda-se um ganho de 10 até no máximo $15 \mathrm{~kg}^{13}$.

É desaconselhável que o ganho de peso em uma gestação exceda, pois este é um dos principais indicativos de alterações osteomusculares, circulatórias, respiratórias e endócrinas. Aumentos repentinos de peso devem ser observados, pois podem ser indicativos de retenção de líquido, TPE (Toxemia Pré-eclâmptica) e dificuldade de desenvolvimento do bebê após o nascimento ${ }^{1,22,23}$.

O papel do fisioterapeuta durante o parto supera as orientações oferecidas no pré-natal, pois possui recursos alternativos para condução deste com o uso das bolas suíças, terapia manual, adoção de posturas verticais e banhos de chuveiro, idealizados no protocolo do $\mathrm{G} 1$, onde se observou que $60 \%$ das gestantes analisadas nesse grupo evoluíram para o parto normal e o tempo de intervenção fisioterapêutica durou em média 165min, sendo que para Freitas (1990), o tempo total do trabalho de parto ativo é em média de 276 min para primíparas e de $144 \mathrm{~min}$ para multíparas. Em comparação entre o G1 e o G2, não foi observada diferença estatisticamente significativa na evolução do parto e tão pouco no tempo de intervenção fisioterapêutica, provavelmente pelo número da amostra, porém, segundo umas das gestantes (Entrevistada 04) "Foi muito bom. Eu gostei, pois me ajudou na evolução do meu parto. Acho que não conseguiria sem essa ajuda. Me ajudou a ficar mais calma" ${ }^{21,24}$.

Para Mamede (2007), fisiologicamente é melhor para a mãe e para o bebê, quando a mulher está acompanhada de um fisioterapeuta durante o trabalho de parto, mantendo-se assistida e em atividade específica, pois o útero contrai-se 


\section{Atenção à Saúde}

com eficácia e o fluxo sanguíneo que chega ao bebê através da placenta é abundante, diminui o período da fase ativa do trabalho de parto e a dor é reduzida ${ }^{25}$. Observou-se que no Centro Obstétrico sede desta pesquisa, o Fisioterapeuta não faz parte da equipe interdisciplinar, mesmo assim o uso de mecanismos que promovem relaxamento e alívio das tensões do trabalho de parto, como o banho de chuveiro e uso da bola suíça, fazem parte da rotina, embora aplicados pelos profissionais ali presentes somente embasados em suas observações empíricas. Porém, é comum o uso de medicamentos que induzem o processo do trabalho de parto, visto que em um grupo 100\% das gestantes receberam ocitocina e no outro $50 \%$ recebeu o misoprostol. Segundo alguns estudos, a presença do Fisioterapeuta e os métodos utilizados retardam o uso de drogas farmacológicas utilizadas para analgesia, bem como os medicamentos que aceleram o processo do trabalho de parto ${ }^{18,26,27,28}$.

O'connor ${ }^{1}$, em revisão técnico-científica, apresenta a inserção interdisciplinar do fisioterapeuta como contribuinte à gestante, firmemente estabelecida em países como Inglaterra, África do Sul, Austrália e Canadá. O profissional fisioterapeuta, no Brasil, ainda não faz parte integral da equipe interdisciplinar na maioria dos centros obstétricos, tanto que, na amostra desse estudo somente 2 gestantes tinham conhecimento sobre os trabalhos da fisioterapia durante o período pré-parto, parto e puerpério, confirmado na fala de uma das gestantes "Achei muito legal, os exercícios são bons e ajudam bastante a aliviar a dor. Não sabia que existia esse tipo de tratamento e achei que ajuda a deixar a mulher mais calma" (Entrevistada 05). Isso demonstra que ainda há um campo de trabalho a ser explorado por esse profissional, e uma fragilidade no serviço de saúde prestado às gestantes ${ }^{01,29}$.

Diante dos resultados evidenciados pelo estudo, essencialmente ao se tratar do G1, a fisioterapia e o papel desempenhado pela intervenção baseada na adoção da terapia manual e de posturas verticais, mostrou-se benéfica para as parturientes quando comparadas ao G2. A literatura demonstra que a inserção do fisioterapeuta em centros obstétricos e a participação ativa nos estágios do parto com uso de recursos como terapia manual e posturais verticais, fazem com que este profissional configure denotada importância e uma maior inserção em centros obstétricos na contemporaneidade ${ }^{4,9,11,18}$. 


\section{CONCLUSÃO}

Mesmo tendo observado que não houve diferença estatisticamente significativa na diminuição da dor entre os grupos, durante a aplicação da EVA percebeu-se a diminuição da dor do $\mathrm{G} 1$ a partir dos 60 minutos de intervenção fisioterapêutica durante o trabalho de parto e pelo relato dos próprios pesquisados. O bem estar físico, juntamente com um aumento da confiança e uma maior consciência do processo de parturição mais ativo, natural e satisfatório, faz parte da assistência fisioterapêutica.

Concluímos que a inserção crescente do profissional fisioterapeuta junto à equipe de Centros Obstétricos justifica-se ao passo que este pode atuar na orientação sobre a mobilidade adequada à gestante, aderindo o uso de posturas verticais bem como o uso de técnicas de terapia manual, influenciando de maneira positiva na fase ativa do trabalho de parto, diminuindo a dor e o tempo do trabalho de parto, mostrando então a relevância desse estudo acerca da investigação da adoção de tais recursos em centros obstétricos.

\section{REFERÊNCIAS}

1. O'connor LJ, Stephenson RG. Fisioterapia Aplicada à ginecologia e obstetrícia. Barueri: Manole; 2004.

2. Souza ELBL. Fisioterapia aplicada à obstetrícia, uroginecologia e aspectos de mastologia. Rio de Janeiro: Guanabara Koogan; 2007.

3. Bavaresco GZ, Souza RSO, Almeica B, Sabatino JH, Dias M. O fisioterapeuta como profissional de suporte à parturiente. Ciência saúde coletiva. 2011;16:3259-66.

4. Souza ELBL. Fisioterapia aplicada à obstetrícia \& aspectos de neonatologia: uma visão multidisciplinar. Belo Horizonte: Health; 1999.

5. Leveno KJ. Manual de obstetrícia de Williams. Porto Alegre: Artmed; 2005.

6. Castro AS, Castro AC, Mendonca AC. Abordagem fisioterapêutica no pré-parto: proposta de protocolo e avaliação da dor. Fisioterapia e Pesquisa. 2012;19:210-14.

7. Santana LS, Galo RBS, Marcolin AC, Quintana SM. Avaliação da intensidade da dor na fase ativa do trabalho de parto em primigestas. Revista Dor. 2010;11:214-17. 
8. Santos TC, Anjos MS. Atuação Fisioterapêutica na Sala de Parto Normal. Fisioterapia Brasil. 2006;7:229-34.

9. Zamataro VO. O papel da fisioterapia no preparo para o parto de cócoras. Fisioterapia em Movimento. 1996;8:48-53.

10. Nakano S, Ana MS. O efeito da deambulação na duração da fase ativa do trabalho de parto. Escola Anna Nery Revista de Enfermagem. 2007;11:466-71.

11. Rett MT, Bernardes NO, Santos AM, Oliveira MR, Andrade SC. Atendimento de puérperas pela fisioterapia em uma maternidade pública humanizada. Fisioterapia e Pesquisa. 2008;15:361-6.

12. Silva APS, Silva JS. A importância dos músculos do assoalho pélvico, sob uma visão anatômica. Fisioterapia Brasil. 2003;4.

13. Organização Mundial de Saúde. (Anais eletrônico) 2014 (Acesso em 02 de maio. 2014). Disponível a partir de: URL: www.who.int.

14. Medronho RA. Epidemiologia. São Paulo: Atheneu; 2004.

15. Torres DFM. Fisioterapia: guia prático para a clínica. Rio de Janeiro: Guanabara Koogan; 2006.

16. Souza FAEF, Pereira LV. Dor: o quinto sinal vital. Revista Latino-am Enfermagem. 2002;10:446-7.

17. Mazzali L, Nascimento GR. Análise do tratamento fisioterapêutico na diminuição da dor durante o trabalho de parto normal. Ensaios e Ciência: Ciências Biológicas, Agrárias e da Saúde 2008XII7-17. Disponível em: http://www.redalyc.org/articulo.oa?id=26012806002. Data de consulta: 31 / mayo / 2014.

18. Santos TC, Anjos MS. Atuação do Fisioterapeuta na sala de parto normal. Revista Fisioterapia Brasil. 2006;7.

19. Abreu EA, Santos JDM, Ventura PL. Efetividade da eletroestimulação nervosa transcutânea no alívio da dor durante o trabalho de parto: um ensaio clínico controlado. Revista Dor. 2010;11:313-18.

20. Brandão ER, Heilborn ML. Sexualidade e gravidez na adolescência entre jovens de camadas médias do Rio de janeiro. Caderno de Saúde Pública. 2006;22:1421-30.

21. Canesin KF, Amaral WN. A atuação fisioterapêutica para diminuição do tempo do trabalho de parto: revisão de literatura. Femina. 2010;38:429-33.

22. Bio E, Bittar RE, Zugaib M. Influência da mobilidade materna na duração da fase ativa do trabalho de parto. Revista Brasileira de Ginecologia e Obstetrícia. 2006;28: 671-79. 
23. Polden M, Mantle J. Fisioterapia em obstetrícia e ginecologia. São Paulo: Livraria Editora; 1997.

24. Freitas F, et al. Rotinas em Obstetrícia. Porto Alegre: Artmed; 2011.

25. Mamede FV, Almeida AM, Souza L, Mamede MV. A Dor Durante O Trabalho De Parto: O Efeito Da Deambulação. Revista Latino-am Enfermagem. 2007;15:446-7.

26. Orange FA, Amorim MMR, Lima L. Uso da eletroestimulação transcutânea para alívio da dor durante o trabalho de parto em uma maternidade-escola: ensaio clínico controlado. Revista Brasileira de Ginecologia e Obstetrícia. 2003;25:45-52.

27. Silva LM, Oliveira SMJV, Silva FMB, Alvarenga MB. Uso da bola suíça no trabalho de parto. Acta Paulista de Enfermagem. 2011;24:656-62.

28. Davim RM, Torres GV, Melo ES. Estratégias não farmacológicas no alívio da dor durante o trabalho de parto: pré-teste de um instrumento. Revista Latino-am de Enfermagem. 2007;15:1150-6.

29. Chaves KRO, Mejia DPM. A influência da cinesioterapia na fase ativa do trabalho de parto. Pós-graduação em Fisioterapia em Urologia, Obstetrícia e Mastologia Faculdade Ávila. (Anais eletrônicos) 2014 (acessado em 31 de maio de 2014). Disponível a partir de: URL: www.portalbiocursos.com.br

30. Berek JS, Novak ER. Berek \& Novak Tratado de Ginecologia. Rio de Janeiro: Guanabara Koogan; 2008. 